\title{
The Application of a Novel, Context Specific, Remote, Usability Assessment Tool to Conduct a Pre-Redesign and Post- Redesign Usability Comparison of a Telemedicine Website
}

\author{
Jessica Lynn CAMPBELL ${ }^{\mathrm{a}, 1}$ and Helen MONKMAN ${ }^{\mathrm{b}}$ \\ ${ }^{a}$ Department of English, University of Central Florida, Orlando, Florida, 32816 \\ ${ }^{\mathrm{b}}$ School of Health Information Science, University of Victoria, Canada
}

\begin{abstract}
The usability of telemedicine provider websites is an essential component of increasing the public's awareness of, understanding of, and safe and effective use of telemedicine. A newly developed usability data collection tool was used to conduct a usability assessment of the Teladoc website pre- and post- a redesign. The results suggest that the Teladoc website had better usability prior to the redesign. The Teladoc Website Usability Survey (TWUS) developed for the Teladoc website usability assessment can be modified and used in future usability studies of telemedicine provider interfaces. Usability research that takes place in specific context of use settings is more valuable to identify usability problems.
\end{abstract}

Keywords. Telemedicine, Health Information Technology, HIT, usability, user interface design, website design, UX, remote usability testing, qualitative research

\section{Introduction}

Telemedicine is an alternative medical care option, whereby medical consultation, diagnosis, and treatment is delivered via telecommunication technologies [1]. Direct-toConsumer (DTC) telemedicine, a specific application of telemedicine for the general public, is the synchronous, real-time delivery of healthcare via remote, digital interfaces [2]. DTC telemedicine enables physicians to consult with patients virtually typically through a video chat online or an app on their smartphone. DTC telemedicine is purported to increase access to healthcare, reduce costs, and be convenient [3].

The usability of telemedicine platforms is essential for patients to be able to safely and effectively interact with a physician and receive quality healthcare [4]. Usability is the extent to which a product can be used by target users to achieve specific goals with effectiveness, efficiency, and satisfaction in their context of use [5]. The delicate nature of healthcare predicates that usability is a critical factor affecting the widespread adoption of healthcare technology by the public [6,7]. Despite the purported benefits of

\footnotetext{
${ }^{1}$ Corresponding Author, Jessica Lynn Campbell, University of Central Florida, Orlando, Florida, USA; E-mail: jessicalynn@embarqmail.com.
} 
telemedicine, DTC telemedicine has received limited uptake and use by the public, especially those who are underserved and/or have limited health literacy [8].

\subsection{Background}

Usability from a widespread, multifaceted population, like the general public, hinges on important design aspects of the user interface, as well as subjective human characteristics, such as an individual's health literacy level [7]. DTC telemedicine is marketed to the public via their website. Thereby, patients first interaction with DTC telemedicine is likely to be the telemedicine provider website. DTC telemedicine websites must have a sufficient level of usability for diverse general public users to become aware of telemedicine, be able to interact with the website easily, and be able to perform the actions required to connect with a doctor virtually. Evaluating the usability of DTC telemedicine websites and being able to apply the insight gained into improving the usability of these health platforms and other similar health information technology (HIT) may ameliorate some of the disparities in healthcare.

\subsection{Scope of Study}

The objective of this study was to demonstrate how a novel usability data collection instrument was created and applied to evaluate the usability of the Teladoc public-facing website prior to and subsequent to a redesign. Remote usability testing was conducted prior to and subsequent to Teladoc Health, Inc. going live with a redesigned website on October 24, 2019 [9]. The methods used in this part were reviewed by the UCF IRB (ID: STUDY00000638) and found to be exempt from any regulations regarding human subjects research. The two usability testing sessions serve as comparable data in which to discover how valuable Teladoc Health, Inc.'s redesign efforts were at meeting patients' usability needs that better equip patients in utilizing the service.

\section{Methods}

Teladoc, one of the largest DTC telemedicine providers [10] was selected as the object of analysis in this study because it is a well-known DTC telemedicine provider and the insight gained can be applied to improve the usability of other telemedicine platforms or similar HITs. Remote usability testing was the approach used to allow subjects to perform in a context in which they would likely seek telemedicine and be able to simulate a real-life interaction with the Teladoc website, for instance, in their home. Results from usability testing in context-specific settings are more indicative of usability problems because subjects are in a real-life context of use [11]. Therefore, subjects are better able to simulate how they would interact with an object or product in a real-life situation, which increases the ability to identify specific usability problems they encounter. Amazon Mechanical Turk was used to be able to recruit a large, heterogeneous population of participants, who best represent the laypeople who are most likely to need and use a telemedicine service [12]. 


\subsection{Selection of Representative Users: Patients}

Fifty participants $(n=50)$ took part in the pre-redesign usability assessment on September 9, 2019. Respondents ranged in age from 23 to 59, with a mean age of 35.6. Most participants ( 84 percent) reported that they were familiar with telemedicine prior to completing the survey, and one participant ( 2 percent) reported to have not been familiar with telemedicine. Fourteen percent of the participants indicated they were unsure of what telemedicine was. Thirty-three participants $(n=33)$ took part in the post-redesign usability assessment on November 5, 2019. Respondents ranged in age from 24 to 60, with a mean age of 35.2. Most participants ( 73 percent) reported that they were familiar with telemedicine prior to completing the survey, and twelve percent reported to have not been familiar with telemedicine. Fifteen percent of the participants indicated they were unsure of what telemedicine was. The smaller number of participants in the postredesign study was due to lack of funds available for recruiting participants.

\subsection{Data Collection Using TWUS}

A novel data collection instrument was developed to be able to perform remote usability testing. The new tool, called Teladoc Website Usability Survey (TWUS), asked participants to perform tasks and activities using the Teladoc website that patients would likely need to in order to perform a virtual doctor. Task completion success was the metric of usability obtained. See Figure 1 for the complete survey. Some questions were task-based (Qs 1-7) and a some were subjective (Qs 8-11). The questions were specifically designed to comprehensively assess several usability qualities, including effectiveness (task completion success) and user perception of use (subjective user feedback). The usability qualities were measured using task completion success rates and qualitative data analysis of subjective user feedback, as both affect usability [13]. The qualitative data analysis of the subjective user responses and the codebook developed that categorized usability improvement suggestions are beyond the scope of this study.

\section{Results}

The TWUS collected task completion success data, which was used to measure usability. The average rate of task completion success for the pre-redesign group was 95 percent. The average rate of task completion success was 94 for the post-redesign group. To recall, this group interacted with the Teladoc website after it had undergone design changes on October 24, 2019. See Figure 1 for complete results.

\section{Discussion}

The Teladoc website subsequent to the redesign appeared to have poorer usability than the previous interface. Several of the participants interacting with the post-redesign Teladoc website failed to be able to execute some of the most critical activities required for people to be able to access healthcare from the Teladoc website, or in other words, perform a virtual doctor visit. For example, several participants in the post-redesign 
group were unable to locate the page that describes how Teladoc works (see Figure 1 Q4) or how to get prescription medications (see Figure 1 Q-3).

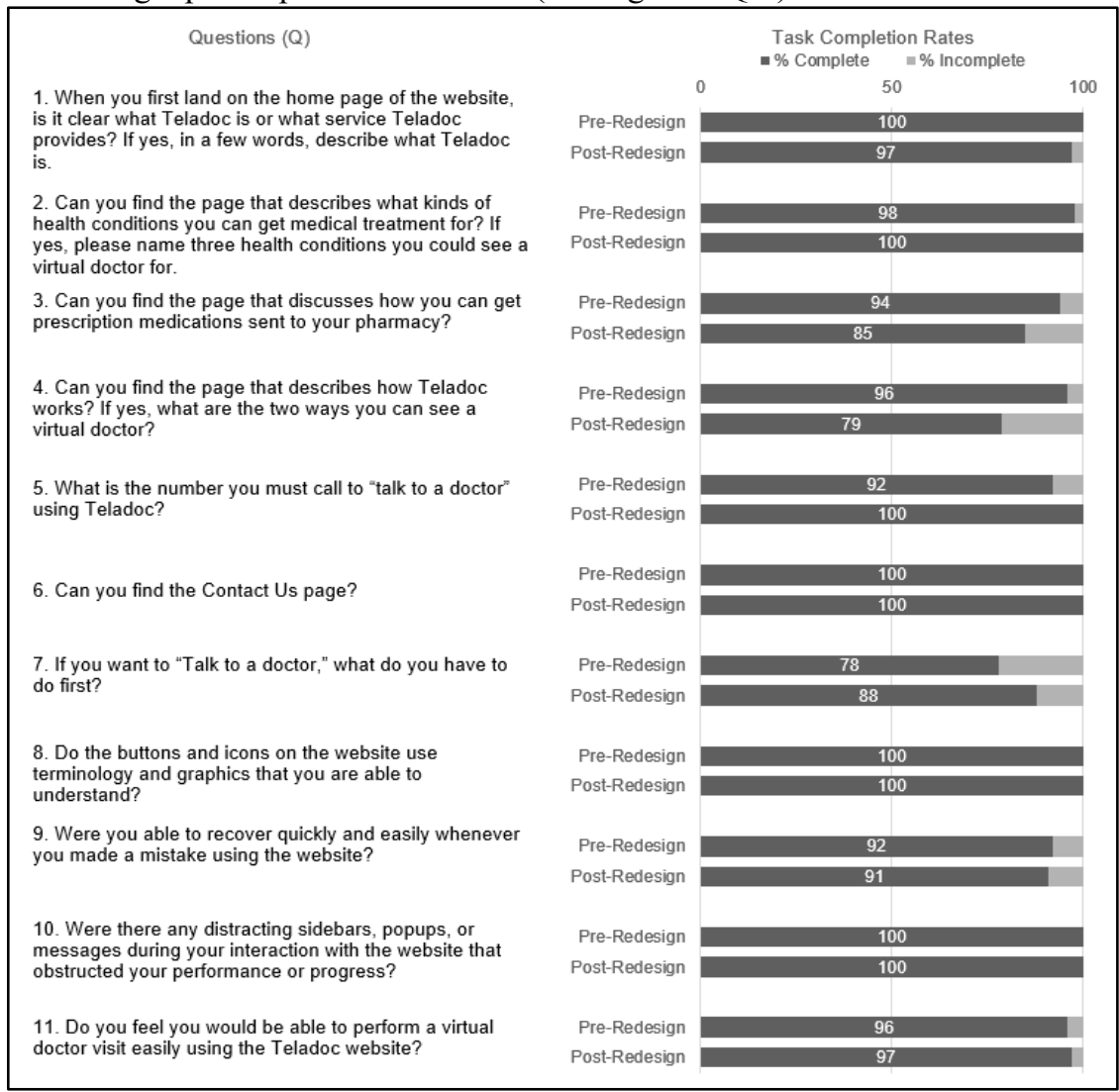

Figure 1. Amazon Mechanical Turk Remote Usability Testing of the Teladoc Website: Task and Completion Success or Failure (\%) Pre- and Post- Website Redesign

However, other critical design aspects of the interface were improved with the redesigned website. Many $(\mathrm{n}=11)$ of the participants prior to the redesign did not know what she or he had to do first in order to "Talk to a doctor." The finding that the task completion success rate for this task improved in the post-redesign group indicates that Teladoc, Inc. may have improved the clarity of this activity or used lay terminology to describe this activity on their post-redesign website. Based on the findings of the preand post- usability testing of the Teladoc website, Teladoc Inc. did not appear to enhance usability when revamping their website. This might suggest that Teladoc Inc., does not perform user testing in order to discover how users interact with the website in ways that enable them to perform a virtual doctor visit.

\section{Conclusions}

In order for patients to be able to leverage telemedicine, they must first be able to use the DTC telemedicine website sufficiently and safely in their time of need. Based on the 
findings of the pre- and post- usability assessment of the Teladoc website, Teladoc Inc. did not appear to enhance usability when redesigning their website. The remote usability testing method and data collection instrument developed for this study can be applied to other HIT usability studies. The TWUS can be modified to be applied to other telemedicine provider interfaces. Researchers are encouraged to develop new techniques to evaluate the usability of consumer health information systems that best suit their particular context of use and target user population. If laypeople are unable to use HIT, the impetus of HIT to increase quality healthcare access will stagnate. Remote usability testing is an effective method to being able to simulate patients' real-life interaction with HIT and the insight gained can be used to optimize the usability of HIT. Teladoc, Inc. and other telemedicine providers can reference this study and improve their public-facing website, which will likely to increase patient acceptance and widespread adoption and diffusion of telemedicine.

\section{Acknowledgements}

This study was funded by a grant from the University of Central Florida College of Graduate Studies and their support is appreciated.

\section{References}

[1] Bashshur RL. On the definition and evaluation of telemedicine. Telemed J. 1995;1(1):19-30.

[2] Uscher-Pines L, Mulcahy A, Cowling D, Hunter G, Burns R, Mehrotra A. Antibiotic Prescribing for Acute Respiratory Infections in Direct-to-Consumer Telemedicine Visits. JAMA Intern Med. 2015 Jul $1 ; 175(7): 1234$.

[3] Uscher-Pines L, Mehrotra A. Analysis of Teladoc Use Seems To Indicate Expanded Access To Care For Patients Without Prior Connection To A Provider. Health Affairs. 2014 Feb 1;33(2):258-64.

[4] Preece J, Sharp H, Rogers Y. Interaction design: beyond human-computer interaction. New York: John Wiley \& Sons, Inc; 2002. p. 519.

[5] ISO 9241-11:2018(en), Ergonomics of human-system interaction - Part 11: Usability: Definitions and concepts [Internet]. Available at: https://www.iso.org/obp/ui/\#iso:std:iso:9241:-11:ed-2:v1:en, Accessed 2021 Jan 13.

[6] Kushniruk A, Borycki E, Kitson N, Kannry J. Development of a Video Coding Scheme Focused on Socio-Technical Aspects of Human-Computer Interaction in Healthcare. Stud Health Technol Inform. 2019;257:236-243.

[7] Monkman H, Kushniruk A. A health literacy and usability heuristic evaluation of a mobile consumer health application. Stud Health Technol Inform. 2013;192:724-8.

[8] Sarkar U, Karter AJ, Liu JY, Adler NE, Nguyen R, Lopez A, Schillinger D. The literacy divide: health literacy and the use of an internet-based patient portal in an integrated health system-results from the diabetes study of northern California (DISTANCE). J Health Commun. 2010;15 Suppl 2:183-96.

[9] Alday AS. (Teladoc Health, Inc.) email to: Jessica Lynn Campbell. 2020 Jan. 7.

[10] Roland J. Telemedicine: 10 Best Companies [Internet]. Available at: https://www.healthline.com/health/best-telemedicine-companies\#1, Accessed 2020 Oct 13.

[11] Kushniruk A, Nohr C, Jensen S, Borycki EM. From Usability Testing to Clinical Simulations: Bringing Context into the Design and Evaluation of Usable and Safe Health Information Technologies. Contribution of the IMIA Human Factors Engineering for Healthcare Informatics Working Group. Yearb Med Inform. 2013;8:78-85.

[12] Monkman H, Kushniruk AW. eHealth literacy issues, constructs, models, and methods for health information technology design and evaluation. Knowledge Management \& E-Learning: An International Journal. 2015;7(4):541-9.

[13] Davis F. Perceived Usefulness, Perceived Ease of Use, and User Acceptance of Information Technology. MIS Quarterly. 1989 13(3):319-40. 